\title{
Moving Beyond Community Mental Health: Public Mental Health as an Emerging Focus for Psychiatry Residency Training
}

\author{
Laura Weiss Roberts • Eugene V. Beresin • \\ John H. Coverdale • Richard Balon • Alan K. Louie • \\ Jane Paik Kim • Maurice M. Ohayon
}

Received: 19 September 2014 / Accepted: 24 September 2014 / Published online: 23 October 2014

(C) Academic Psychiatry 2014

Mental disorders and behavioral conditions represent a challenge to the well-being of communities throughout the world. This statement is bold, certainly, but not without basis. One in four adults in the USA meets criteria for a mental disorder, and estimates from the World Health Organization indicate that mental disorders represent nearly $23 \%$ of years lived with disability for all people globally $[1,2]$. This level of disease burden is greater than that associated with HIV/AIDS, cancer, and diabetes combined. Moreover, the most common causes of death, including ischemic heart disease, stroke, chronic obstructive pulmonary disease, diabetes, HIV, and multiple forms of cancer, are linked with at-risk behaviors and behavioral conditions. The number of completed suicides each yearnearly 900,000 worldwide and 37,000 in the USA - in rough terms represents more than twice the number of people who die annually from war or from interpersonal violence.

By 2030, depression will be the top cause of disease burden globally, 1.01 million people will die from self-inflicted injury each year, and 1.85 million people will die from traffic accidents frequently associated with substance-related disorders, sleep deprivation, and co-occurring physical and mental health conditions [2]. The burden associated with mental illness and behavioral disorders will continue to be the greatest among certain vulnerable subpopulations, including

L. W. Roberts $(\bowtie) \cdot$ A. K. Louie · J. P. Kim • M. M. Ohayon Stanford University School of Medicine, Stanford, CA, USA e-mail: RobertsL@stanford.edu

E. V. Beresin

Harvard Medical School, Boston, MA, USA

J. H. Coverdale

Baylor College of Medicine, Houston, TX, USA

R. Balon

Wayne State University, Detroit, MI, USA socioeconomically disadvantaged children, certain underrepresented minorities, the homeless, rural and immigrant populations, and individuals with co-occurring, stigmatized, and severe physical illnesses. For these reasons, the impact of mental disorders and behavioral conditions can be characterized as one of the greatest concerns of our world.

Mental disorders are the largest diagnostic category for those in the USA receiving disability, at a cost of $\$ 25$ billion a year [3]. Even with decades of research, antipsychotic medication, and deinstitutionalization, evidence of improved prospects for recovery remains scarce. Of those with schizophrenia, $80 \%$ are unemployed; $60 \%$ of individuals with bipolar disorder experience co-occurring substance abuse dependency; posttraumatic stress disorder is not less prevalent today than it was 50 years ago [3]. Together, these outcomes data demonstrate a concerning, sobering unmet need in public mental health.

Are we doing enough in residency training to prepare future psychiatrists to address these grave realities? Do residency curricula offer sufficient exposure to the range of issues faced by the diverse communities and distinctly vulnerable subpopulations hardest hit by mental illness and related health conditions? Further, are we providing physicians-in-training - those who will be established in their careers by 2030 - with the means to improve population mental health, as well as to enhance the well-being and resilience of their individual patients? Are we giving the field of psychiatry and our patients, as well as our physicians-in-training, a reason to be hopeful in light of the sobering facts on state of mental health?

\section{What Is Public Mental Health?}

Public mental health can be understood as a multidisciplinary field dealing with the prevention, identification, evaluation, treatment, and management of mental disorders on a large 
human scale and engaging in activities to promote well-being across and within populations. Leaders in public mental health seek to reduce health disparities through systemic efforts at local, regional, national, and international levels. Evidence derived from researchers in this field help to inform legislative actions, advocacy, and population-based educational and destigmatization programs, along with programs to reduce violence and suicide. Challenges undertaken by public mental health include broad, population-based endeavors to identify risk factors for mental disorders; to enhance resilience of all individuals, including those most at-risk for health issues; to compare and enhance the effectiveness of interventions targeting mental comorbidities; to improve access to care particularly among underserved populations; to provide funding and training for mental disorders; to survey the prevalence of mental disorders and their medical comorbidities, treatments, and outcomes; to personalize care for patients on the basis of their individual responses; and to improve the outcomes of patients who suffer from disorders. Professionals who are in the field of public mental health may perform work (e.g., consultations, policy advisory activities) that seek to strengthen and shape activities in contexts that are outside traditional medical settings, such as schools, courts, communities, and broad systems or networks that are responsible for large numbers of individuals and families with mental illness. Ethics, professionalism, and social policy considerations that pertain to health disparities and vulnerability are highly salient to the field of public mental health. Moreover, the scope of public mental health is broader than a focus on "diseases" to include efforts to study and optimize the quality of life experienced in communities and the overall well-being of people who belong to distinct groups, subpopulations, and large populations.

The field of public mental health is thus linked with global mental health and community mental health. Global mental health addresses social ills and other factors such as climate change that might contribute to mental health and illness across international settings. Global mental health expands to include prevention, intervention, education, and improvements in the awareness of and care for mentally ill individuals in nations around the world. The major effort in global mental health has been to help countries with the greatest burden of mental illness and the most severe lack of resources. Community mental health may be conceptualized as falling under the broad rubric of public mental health and as focusing particularly on the early recognition and evidence-based treatment of individuals with acute and chronic major mental disorders in community settings. These settings are usually outpatient sites such as community mental health centers or medical clinics integrated with psychiatric clinics in rural or urban environments, but they could also include incarcerated populations, schools, and other local groups and organizations. Community mental health services include medication clinics and comprehensive psychosocial interventions. Although the latter has received relatively less attention in comparison with its psychopharmacological counterparts, evidence has been rather encouraging for individuals with schizophrenia and mood and anxiety disorders. Many studies have shown the effectiveness of interventions such as community programs, family psychoeducation, yet few patients actually receive evidence-based psychosocial treatments $[4,5]$. These can include local prevention efforts across medical disciplines, including early warning sign management, housing provisions, benefits and opportunities for vocational rehabilitation, advocacy and education programs, and initiatives to identify unmet needs and to integrate and coordinate care at community levels.

Psychiatric epidemiology and biostatistics together represent a basic science of public mental health. These quantitative disciplines provide rigorous methods of inquiry and analysis that can be applied to improve outcomes, enhance understanding of wellness, mental health and illness at global, public health, and community levels, and implement effective interventions. Other forms of study linked with the social sciences contribute to knowledge of distinct groups and entire populations. Examples may include qualitative studies of the lived experiences of mentally ill homeless people, services research that assesses health outcomes in the context of certain systems of care, studies of rural and urban health disparities, and crossdisciplinary studies of social policy and mental health outcomes. Studies such as these help pave the way for greater knowledge regarding the prevention and recovery from illnesses.

\section{Public Mental Health and General Residency Training}

The field of public mental health is of immense importance because it seeks to promote the health of entire populations through efforts to prevent and treat mental disorders and to foster well-being and overall quality of life. This said, curricular attention to these topics in general psychiatry residency training has been heterogeneous. In many departments, teaching about the mental health of the public has been more "a labor of love" than response to an educational imperative. We suggest that public mental health has been underappreciated and, further, in light of its true relevance, has been comparatively neglected in psychiatry training.

A careful look at past US residency training guidelines indeed suggests that there have been few requirements to ensure that early-career colleagues learn about public mental health, the impact of mental health at a population level, or population sciences. Community psychiatry and global mental health topics have also received little emphasis historically. In a welcome move, however, newer guidelines (the "Milestones Project") in the USA, as described below, give greater weight to the mental health of the public, as do guidelines drawn, for example, from other countries, such as Canada. 


\section{Previous ACGME Requirements}

The 2007 Program Requirements for Graduate Medical Education [6] from the Accreditation Council for Graduate Medical Education (ACGME) give limited attention to population health, epidemiology, and prevention considerations, and no mention of international health concerns, although an update is due in 2016. The strongest statement of the imperative for public mental health is in the "Medical Knowledge" requirements section [6, p. 19], in which it is stated that the curriculum must cover "the fundamental principles of epidemiology, etiologies, diagnosis, treatment, and prevention of all major psychiatric disorders in the current standard diagnostic statistical manual, including the biological, psychological, sociocultural, and iatrogenic factors that affect the prevention, incidence, prevalence and long term course and treatment of psychiatric disorders and conditions." The program requirements include clinical experiences that "expose" residents to community psychiatry. Although training in community psychiatry is not, strictly speaking, part of public mental health, it does involve some of our most vulnerable and underserved populations and delivery of care in the public sector. This requirement does not stipulate a length of time, and thus some programs may interpret "exposure" liberally.

Other statements, generously interpreted, in the ACGME guidelines appearing in the Common Program Requirements offer some direction regarding the value of including public mental health considerations in residency training. For instance, in Section IV.A.5.a).(1), addressing patient care requirements, the ACGME states that psychiatry residencies must prepare trainees to "be able to provide patient care that is compassionate, appropriate, and effective for the treatment of health problems and the promotion of health" [6, p. 11], including psychiatric health issues, as well as "recognizing and appropriately responding to family violence . . . and its effect on both victims and perpetrators" [6, p. 12]. Later, the role of curricula in imparting and ensuring an understanding of "American culture and subcultures" [6, p. 13] and "research literacy" [6, p. 23] is identified as essential to the competency of a psychiatric trainee. In the competence domain of interpersonal and communication skills, residents' competence in communication related to the health of "the public, as appropriate, across a broad range of socioeconomic and cultural backgrounds" is specified [6, pp. 14, 15]. The section on Professionalism has an explicit requirement for "accountability to patients, society, and the profession" [6, p. 22], and in the section on systems-based practice, the requirements cover "considerations of cost awareness and risk-benefit analysis in patient and/or population-based care as appropriate" [6, p. 16], "work with health care managers and health care providers to assess, coordinate, and improve health care, particularly as it relates to access to mental health care" [3, p. 16], and knowing "how to advocate for the promotion of mental health and the prevention of disease" [6, pp. 16, 17]. More recently, the 2013 "Impact Statement" on the revisions to the ACGME program requirements for psychiatry has no enhancements to the previous essentials of training with respect to public mental health or global psychiatry or related topics. The changes proposed place greater emphasis on the demonstration of "competency" and "proficiency" as trainees progress through their graduate education, rather than time-based curricular experiences.

ACGME general guidelines are currently being updated, and it is reported that they will be released in 2016. The requirements regarding public health and population sciences and the key domains for psychiatry to be articulated in these much-anticipated guidelines are not yet known.

\section{New US Psychiatry Training Requirements}

The Psychiatry Milestones Project [7-14] devised milestones in all of the six ACGME competencies to assist psychiatry residencies in more granular assessments and tracking of residents in their development and progress. The project was an immense undertaking that now provides many more details about training requirements, some of which relate to public mental health. Milestones are organized according to subcompetencies. (In what follows, the name of a subcompetency is followed by its abbreviation in parentheses.) Examples of subcompetencies that touch upon public mental health have been grouped, for simplicity, into four categories: population science; policy/regulations/advocacy; community psychiatry; and systems of care and quality improvement.

1. The first category, population science, most obviously touches on public mental health. The psychopathology subcompetency (MK2) includes the epidemiology of psychiatric disorders over the life cycle and as seen in diverse populations. One of the subcompetencies of professionalism (PROF1) addresses the ability to work with diverse populations.

2. A second category groups together policy, regulations, and advocacy, all common public health areas. The subcompetency named practice of psychiatry (MK6) includes knowledge of policies and regulations that govern mandated reporting, involuntary treatment, and professional standards that are germane to public safety and health. One milestone here includes international differences in regulations. The subcompetency called resource management (SBP2) covers allocation of health resources, cost-effectiveness, and health disparities. Advocacy is found, for instance, in these two subcompetencies (MK6, SBP2) and in the subcompetencies of professionalism (PROF2), systems-based practice (SBP3), and medical knowledge (MK3). 
3. The third category is community psychiatry, associated with many public mental health issues. Communitybased care (SBP3) is a subcompetency including vulnerable and underserved populations, public sector systems of care, prevention, and recovery-based programs. One might also include public education from the teaching subcompetency (PBLI3).

4. Fourth is the category of systems of care and quality improvement that uses some public health principles. The subcompetency termed patient safety and the health care team (SBP1) focuses on systems problems leading to errors. The subcompetency named formal practice-based quality improvement based on established and accepted methodologies (PBLI2) covers quality improvement and projects that improve outcomes in populations.

In sum, as illustrated by the examples across the subcompetencies listed above, one will find many milestones that relate directly or indirectly to public mental health. We commend these efforts to highlight competencies that address the well-being and mental health of groups of individuals and populations. As the Milestones Project unfolds, we will learn more about how residents can and do demonstrate skills and strengths in these domains.

\section{"CanMEDS" Guidelines}

Canadian guidelines, as specified in The Royal College of Physicians and Surgeons' "Objectives of Training in Psychiatry" [15], are more robust in their call for demonstrable competence related to the set of knowledge and skills of importance to the mental health of populations. The "CanMEDS" approach explicitly emphasizes the diverse roles that physicians fulfill in society, including medical expert, communicator, collaborator, manager, health advocate, scholar, and professional. Knowledge and skills in public mental health, population health, or global health are identified as essential for the psychiatrist in training and in practice across many of these roles. For example, as a medical expert, psychiatrists in the CanMEDS system must demonstrate a "working knowledge" of "public health principles" $[15$, p. 5], and as a health advocate, the psychiatrist must be able to "identify the determinants of mental health for the populations that they serve" and "promote the health of . . . populations," including the identification of barriers to care, the discernment of vulnerable or marginalized populations, the ability to describe how public policy affects the health of populations and identification of "points of influence in the health care system and its structure," among other topics $[15$, pp. 12 , 13]. Interestingly, despite their relative strength in focusing on special populations, the CanMEDS guidelines remain relatively silent on the topic of global mental health.

\section{Advancing Public Mental Health}

In this issue of Academic Psychiatry, four pieces highlight public mental health preparation for general residents and advanced trainees in psychiatry. Steiner et al. [16] provide an interesting and valuable overview of the evolution of public psychiatry fellowship programs over the past decade. The authors comment on the diversity of programs, including those with multiple funding sources (e.g., Columbia University) and single funding sources (e.g., Yale University and University of California San Francisco), as well as programs that are funded by grants (e.g., University of Alabama and University of California San Diego) and that seek to prepare psychiatrists for work within the public sector. Greater collaboration, communication, and coordination were seen as essential for the continued success of these programs. In a very exciting contribution, Mangurian, Shumway, and Dilley [17] describe the role that their program has in facilitating the mental health services research preparation of their public psychiatry fellows. The authors describe salutary outcomes with respect to the recruitment of fellows; the revenues generated by fellows, which allow for independence and sustainability of the program; the services research that has been conducted by trainees; and the leadership positions successfully attained by graduates of the fellowship. The authors emphasize the link between clinical and research achievements in their program, and they further comment on the importance of scientific literacy: "although most of these leaders will not become researchers, familiarity with research methods can facilitate quality improvement projects that will help improve care for the vulnerable populations served in the public mental health sector."

In terms of residency training on public mental health topics, Le Melle, Clemmey, and Ranz [18] describe an integrated longitudinal training effort in public psychiatry, focusing primarily on a postgraduate-year 3 curriculum involving experiential and didactic components. A strength of the program related to systems-based practice learning goals is its emphasis on the importance of public psychiatry training in better preparing "residents to navigate the rapidly evolving health care landscape." Referencing the impact of the Affordable Care Act and its relevance for psychiatric education, Khan [19] describes in a letter to the editor findings of a pilot study involving residents in one program. Two-thirds of the resident participants in this voluntary, anonymous study expressed substantial interest in public mental health training. Residents expressed interest in diverse topics, such as school-based mental health, community health centers, foster care and adoption, and global and refugee mental health. Clinical and systems issues pertaining to military, veteran, homeless, incarcerated, and other vulnerable populations were of interest to the respondents. 


\section{Looking Ahead}

Although public mental health covers a wide range of knowledge, skills, and attitudes in a number of academic and clinical domains, we suggest that it will be valuable to consider, along with the authors of these four contributions to our journal, what should be taught and how this teaching should occur in order to help new psychiatry residency graduates promote well-being across groups and populations more effectively. Not only must we consider the content of curricula but also how our teaching efforts and residents' educational activities should evolve under the current healthcare reform movements in the USA. Under the Affordable Care Act, for instance, we shall be seeing an increasing focus on medical homes in the community, with the locus of care shifting from tertiary teaching hospitals to community centers that are increasingly team based. Concomitantly, our need to appreciate principles of population management in practice as our institutions transform into Accountable Care Organizations will similarly increase.

It may be helpful to consider what elements should be present in current required experiences in community psychiatry as a core part of residency training. In such rotations, residents should learn about the promotion and protection of mental health in community settings. Further, they should learn principles of prevention, screening, early detection, and intervention in vulnerable populations. Some of this work necessitates integrating child and adolescent psychiatry into the community rotations, because most psychiatric disorders begin in childhood and in transitional age youth (ages 14-26 years). This component is already offered in many programs.

As mental health advocates and educators in team structures, residents could foster integrated care, including educating and consulting to allied health professionals and staff in schools, courts, and parent groups. Further, they could provide public educational missions in local talks, seminars, and groups within the community. Residents will have to appreciate the cultural groups with whom they are working, including their beliefs and values. Finally, a major component of a community rotation within the scope of public psychiatry should be working to destigmatize mental illness among both health professionals and the public. Supplemental education in community rotations should include learning the principles of population management, epidemiology, global mental health, cross-cultural psychiatry, professionalism and ethics in the care of special populations, principles of advocacy, public policy, and the fiscal underpinnings of the healthcare system. These areas should be taught in didactic seminars, in conjunction with a community clinical rotation.

A new model for teaching public psychiatry would thus build opportunities for progress by including the following strategic objectives: (1) increased formalized training during residency about public mental health, largely through required core community psychiatry rotations; (2) developing model didactic curricula that would supplement clinical rotations, including evidence-based treatments relevant to public health and community mental health; public policy; principles of advocacy; epidemiology; biostatistics; principles of population management, cultural psychiatry, professionalism and ethics, and global mental health; and the fiscal basis for Accountable Care Organizations; (3) developing advanced tracks in residencies and fellowships in public mental health; (4) promoting more academic-community partnerships; and (5), most importantly, increasing monetary incentives for graduates to go into public mental health (e.g., higher salaries, loan-forgiveness plans).

Additionally, we suggest that measures should be taken to encourage more residents to pursue subspecialty fellowship training. This advanced training may occur not only in public psychiatry fellowships (of which there are a handful in the USA) but also in fellowships in addictions, forensics, geriatrics, child and adolescent psychiatry, and neuropsychiatry, because these subspecialties often see populations that are underserved. Advanced training for the care of individuals in rural and frontier settings and in international settings is also desirable, as is training that prepares psychiatrists to conduct epidemiological and related population and health services research.

Resources devoted to the mental health of populations are not sufficient to meet the challenges faced by communities and entire nations throughout the world. From a financial perspective, according to a major report by the US Department of Health and Human Services [20], about $7 \%$ of the total healthcare expenditure was devoted to the treatment psychiatric illnesses. Only 40-50\% of individuals with severe mental illnesses received treatment in the previous year in the USA, however. Two-thirds of these treatments are considered inadequate by the American Psychiatric Association guidelines [21], showing that there is still considerable work to do in adequately training mental healthcare providers. Indeed, these concerns, and others, are far greater in many, perhaps most, countries throughout the world. Also of concern is the wide incongruence between recent increased biological knowledge and the state of mental health care: despite the many surges in basic biological areas of research such as neuroscience, imaging, and genomics, these research advances have not yet translated to realm of public health impact [3]. Although there are many reasons for this gap and the lack of adequate treatment, such as stigma, shortage of psychiatrists, poor access to care, and difficulties navigating a complex and insufficient healthcare system, we suggest that focusing educational resources toward public mental health is critical at this time. Psychiatry residency programs can certainly do more and much to prepare our early career colleagues for the responsibilities they will shoulder in the care of people and populations in the years to come. 
Acknowledgments The authors gratefully acknowledge the editing assistance of Gabrielle Termuehlen and Ann Tennier.

Disclosures The authors have no conflicts of interest.

\section{References}

1. Kessler RC, Chiu WT, Demler O, Merikangas KR, Walters EE. Prevalence, severity, and comorbidity of 12-month DSM-IV disorders in the National Comorbidity Survey Replication. Arch Gen Psychiatry. 2005;62(6):617-27.

2. Whiteford HA, Degenhardt L, Rehm J, Baxter AJ, Ferrari AJ, Erskine HE, et al. Global burden of disease attributable to mental and substance use disorders: findings from the Global Burden of Disease Study 2010. Lancet. 2013;382(9904):1575-86.

3. Insel TR. Translating scientific opportunity into public health impact. Arch Gen Psychiatry. 2009;66(2):128-33.

4. Pharoah F, Mari J, Rathbone J, Wong W. Family intervention for schizophrenia. Cochrance Database Syst Rev. 2006;4, CD000088.

5. President's New Freedom Commission on Mental Health, Achieving the promise: transforming mental health care in America. Final Report. DHHS publication No SMA-03-3832.2003. http://www. mentalhealthcommission.gov/reports/FinalReport/downloads/ FinalReport.pdf. March 17. 2008.

6. Accreditation Council for Graduate Medical Education: ACGME Common Program Requirements, 2007. Available at https://www. acgme.org/acgmeweb/Portals/0/PFAssets/ProgramRequirements/ 400 psychiatry 07012014.pdf. Accessed 19 Sept 2014.

7. Accreditation Council for Graduate Medical Education. The Psychiatry Milestone Project, A Joint Initiative of The Accreditation Council for Graduate Medical Education and The American Board of Psychiatry and Neurology, November 2013. Available at https://www.acgme.org/acgmeweb/Portals/0/PDFs/ Milestones/PsychiatryMilestones.pdf. Accessed 14 Aug 2014.

8. Thomas CR, Keepers G. The milestones for general psychiatry residence training. Acad Psychiatry. 2014;38(3):255-60.

9. Hunt JI, Thomas CR. ACGME Milestone development in general psychiatry: patient care and medical knowledge. Acad Psychiatry. 2014;38(3):261-7.
10. Sanders K, Servis M, Boland R. The four general competencies. Acad Psychiatry. 2014;38(3):268-74.

11. Benjamin S, Widge A, Shaw K. Neuropsychiatry and neuroscience milestones for general psychiatry trainees. Acad Psychiatry. 2014;38(3):275-82.

12. Rosen DE. The development of outpatient milestones. Acad Psychiatry. 2014;38(3):283-7.

13. Widge AS, Hunt J, Servis M. Systems-based practice and practicebased learning for the general psychiatrist: old competencies, new emphasis. Acad Psychiatry. 2014;38(3):288-93.

14. Swing SR, Cowley DS, Bentman A. Assessing resident performance on the psychiatry milestones. Acad Psychiatry. 2014;38(3):294-302.

15. Royal College of Physicians and Surgeons of Canada. Objectives of training in psychiatry, 2009, Editorial Revisions 2014, Version 1.3. Available at http://www.royalcollege.ca/cs/groups/public/documents/ document/y2vk/mdaw/ edisp/tztest3rcpsced000935.pdf. Accessed 15 Sept 2014.

16. Steiner JL, Giggie MA, Koh S, Mangurian C, Ranz JM. The evolution of public psychiatry fellowships. Acad Psychiatry. 2014; 38(6). doi:10.1007/s40596-014-0168-x.

17. Mangurian C, Shumway M, Dilley J. Mental health services research training for the next generation of leaders in the public health sector: a case study of the UCSF/SFGH Public Psychiatry Fellowship. Acad Psychiatry. 2014; 38(6). doi:10. 1007/s40596-014-0040-z.

18. Le Melle S, Clemmey P, Ranz J. Outpatient training in public/ community psychiatry and systems-based practices. Acad Psychiatry. 2014; 38(6). doi:10.1007/s40596-014-0084-0.

19. Khan CT. Trainee interest in public psychiatry amidst implementation of the affordable care act. Acad Psychiatry. 2014; 38(6). doi:10. 1007/s40596-014-0155-2.

20. US Department of Health and Human Services. Organizing and financing mental health services. In: Mental health: a report of the surgeon general. US Department of Health and Human Services, Substance Abuse and Mental Health Services Administration, Center for Mental Health Services, NIH, National Institute of Mental Health, Rockville, MD, USA, 1999, pp. 405-433.

21. Wang PS, Lane M, Olfson M, Pincus HA, Wells KB, Kessler RC. Twelve-month use of mental health services in the United States. Results from the National Comorbidity Survey Replication. Arch Gen Psychiatry. 2005;62(6):629-40. 